\title{
Cystic Meningioma in the Inter-Hemisferic Space Location
}

\author{
Muhammad Zafrullah Arifin, Firman Priguna Tjahjono, Agung Budi Sutiono, Ahmad Faried \\ Department of Neurosurgery, Faculty of Medicine, Universitas Padjdajaran-Dr. Hasan Sadikin General Hospital
}

\begin{abstract}
Objective: The presentation of cystic meningioma in the inter-hemispheric near the falx cerebri is uncommon. It is difficult to differentiate it from intraaxial tumors, such as gliomas. Therefore, it is likely that it is misdiagnosed as other types of brain tumors.
\end{abstract}

Methods: In this study, we reported a cystic meningioma case in the interhemispheric location, showing an intramural nodule on magnetic resonance imaging scans.

Results: Patient underwent surgical treatment and pathological section confirmation revealing meningioma. The patient was a middle-age woman and had been misdiagnosed as suffering from glioma followed by slight hemipharesis on the right extremities.

Conclusions: Although this is a rare case, it will be good if we always consider Received: June 24, 2015 cystic meningioma in inter-hemispheric space when diagnosing this type of cystic lesion if the magnetic resonance imaging (MRI) shows a cystic lesion mimicking glioma image presentation.

Revised:

October 10, 2015

Keywords: Cystic meningioma, interhemispheric space, magnetic resonance imaging, diagnosis

Accepted:

February 26, 2016 IJIHS. 2016;4(1):38-42

\section{Introduction}

There are few case reports available on cystic meningioma. ${ }^{1-7}$ Cystic meningioma is an uncommon variant of menin 7 gioma. This disease is often difficult to distinguish from other intra axial tumors, including necrotic gliomas. Jorge et al. ${ }^{4}$ reported that the cystic meningioma simulates an arachnoid cyst on computed tomography (CT) scan and magnetic resonance imaging (MRI), while Qiu et $a .^{7}$ also reported the presentation of lateral ventricular cystic meningioma. However, cystic meningiomas located in the interhemispheric space are particularly rare and may be difficult to distinguish from other brain tumors. From all types of meningiomas of the central nervous system (CNS), cystic meningiomas are a distinct histological variant of meningiomas accounting for up to $1.6 \%$ of all CNS meningiomas.

Correspondence:

Agung Budi Sutiono, Department of Neurosurgery,

Faculty of Medicine, Universitas Padjdajaran-Dr. Hasan

Sadikin General Hospital

Jl. Pasteur No. 38, Bandung, Indonesia

e-mail: agungbudis@gmail.com
This present case study describes a case of interhemispheric meningiomas exhibiting intratumoral or peritumoral cystic with MRI. Therefore, the purpose of this report was to emphasize that the cystic lesion might have different histological findings, such as in meningiomas.

\section{Case}

A case of 57 years-old female with weakness on the right extremities for one month followed by intermittent headache for the past three months is presented. Neurological examinations revealed hemiparesis of right motor with increased physiology reflex and also pathologic reflex. General examination showed no abnormality. CT-scan was then performed, identifying the presence of an isohypodense mass in the left frontal, with peritumoral edema. This tumor was compressing sulcy and giry. Ventricle and sylvian fissures were also compressed. Cistern was opened and the midline had shifted more than $5 \mathrm{~mm}$ to the right (Fig. 1). The magnetic 


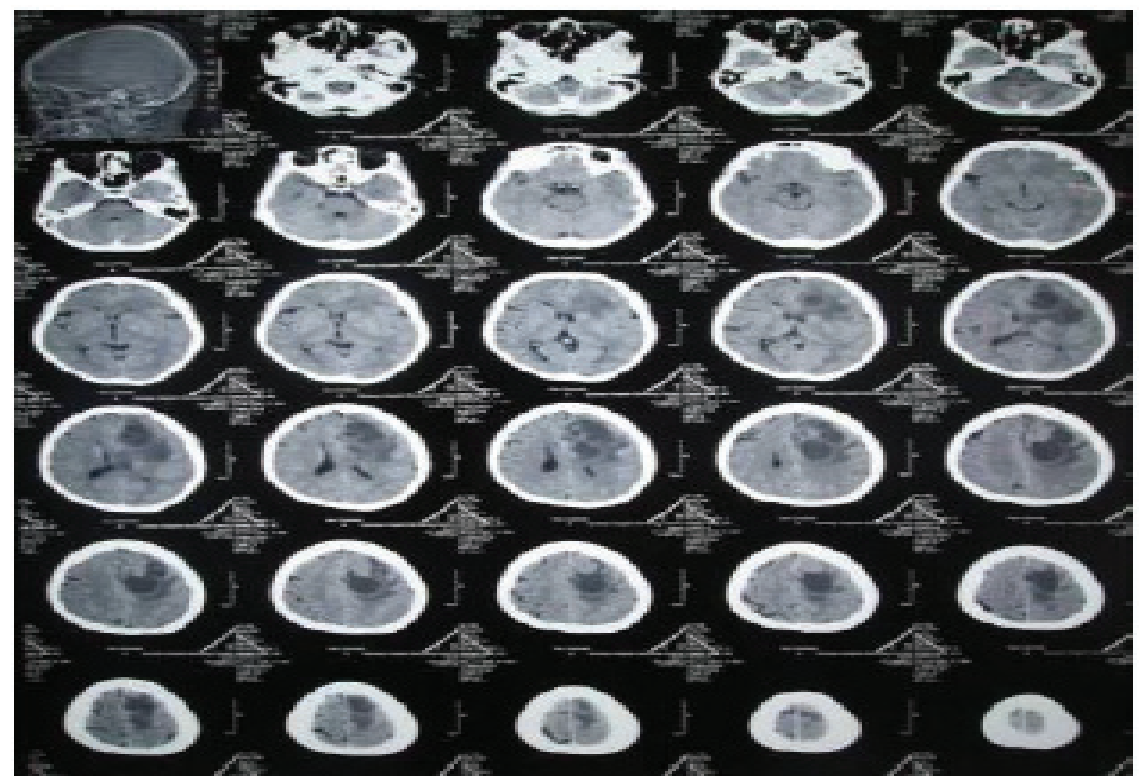

Fig. 1 Isohypodense Mass in Left Frontal, with Peritumoral Edema. Sulcy, Giry, Ventricle, and Sylvian Fissure was Compressed. Cistern was Opened and Midline had Shifted more than $5 \mathrm{Mm}$ to the Right

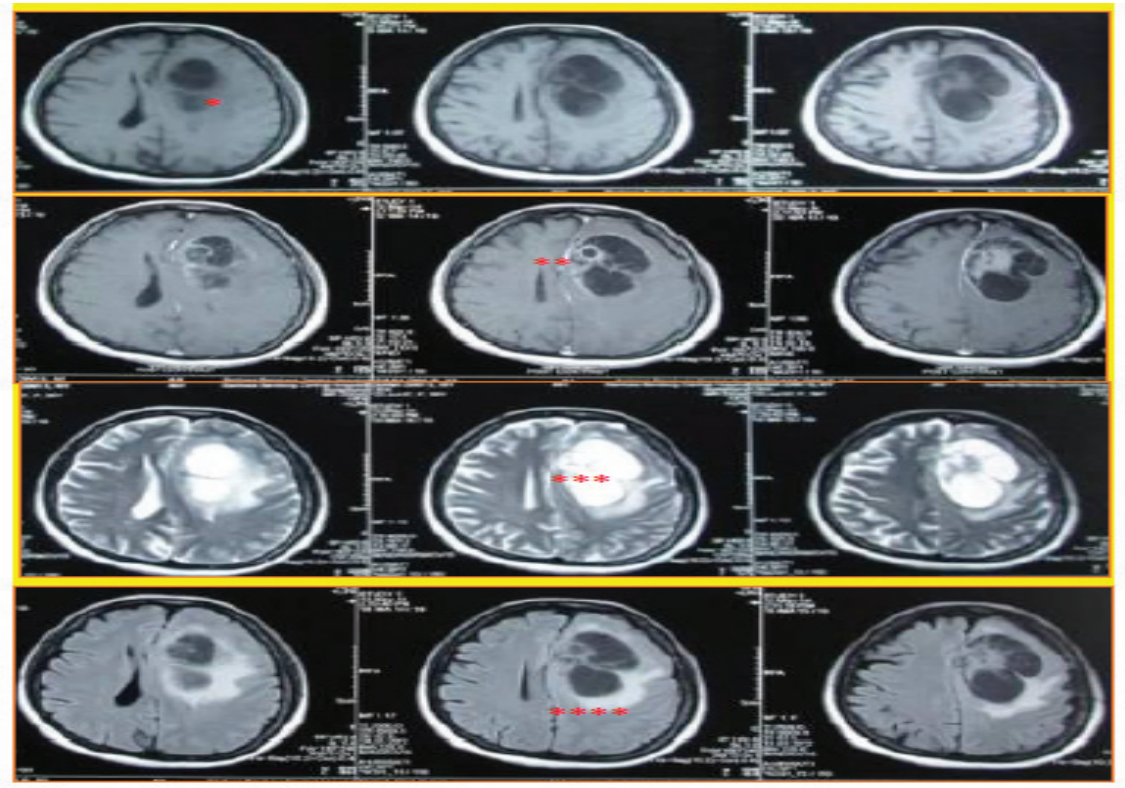

Fig. 2 Isohypointense Mass in Left Frontal on T1 (*). Inhomogenously Enhanced with Positif Ring Enhanced on T1 with Contrast (**). Hyperintense Mass in Left Frontal on T2 (***) with Peritumoral Edema ${ }^{* * * *}$ ) 


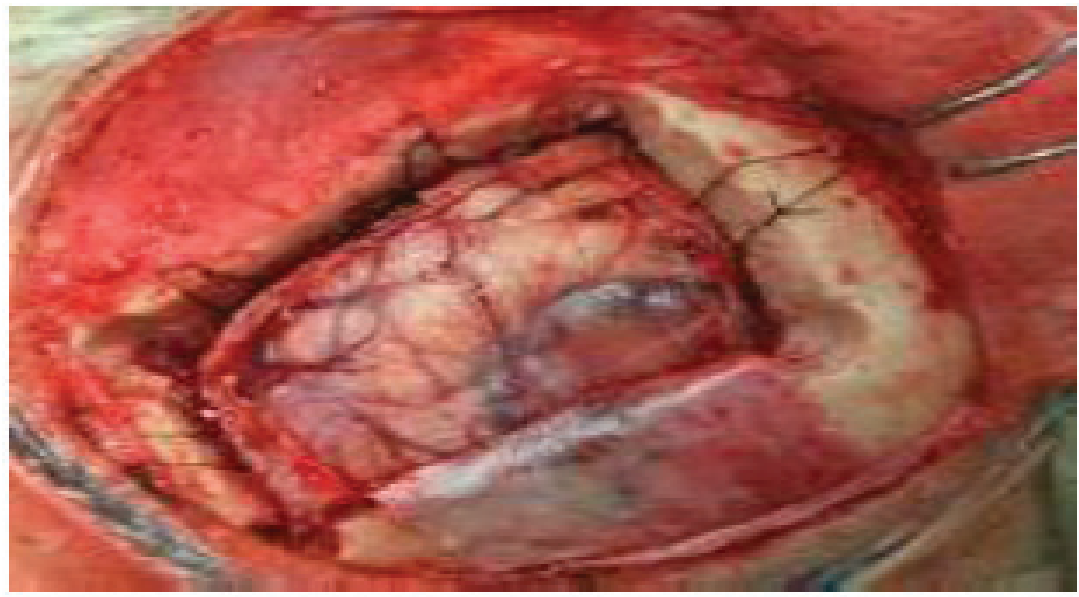

Fig. 3 Mass Located in Interhemispheric Space

resonance imaging (MRI) confirmed this condition (Fig. 2). With these results, the tumor was then diagnosed as a space occupying lession (SOL) supratentorial in left frontal due to suspected high grade glioma. Craniotomy was performed under general anesthesia. Additionally, at the time of surgery, a mass located in interhemispheric space (Fig. 3) was completely resected (Fig. 4).

The pathologic specimen was submitted for microscopic analysis. The macroscopic features showed a cystic lesion containing a dense proteinaceous liquid (Fig. 5). The confirmation of pathological section revealed that it was meningioma (Fig. 6).

\section{Discussion}

Meningiomas are generally solid tumors, and their classical appearance on CT and MRI usually leads to a correct diagnosis. ${ }^{8}$ Mittal et $a .^{5}$ mentioned that meningiomas generally have the characteristic imaging appearance of a well circumscribed, solid, homogeneously enchancing extra-axial mass on both CT and MRI.

A problem with the diagnosis arises when meningiomas have cystic components that can be confused with other tumors, including glial or metastatic tumors with cystic or necrotic

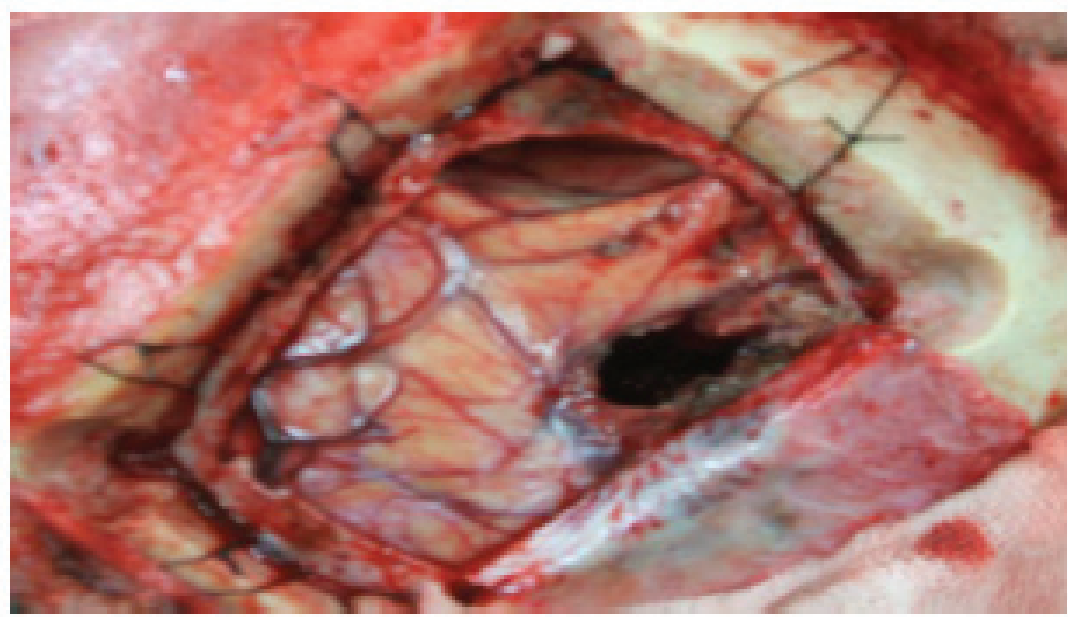

Fig. 4 Mass Completely Resected 


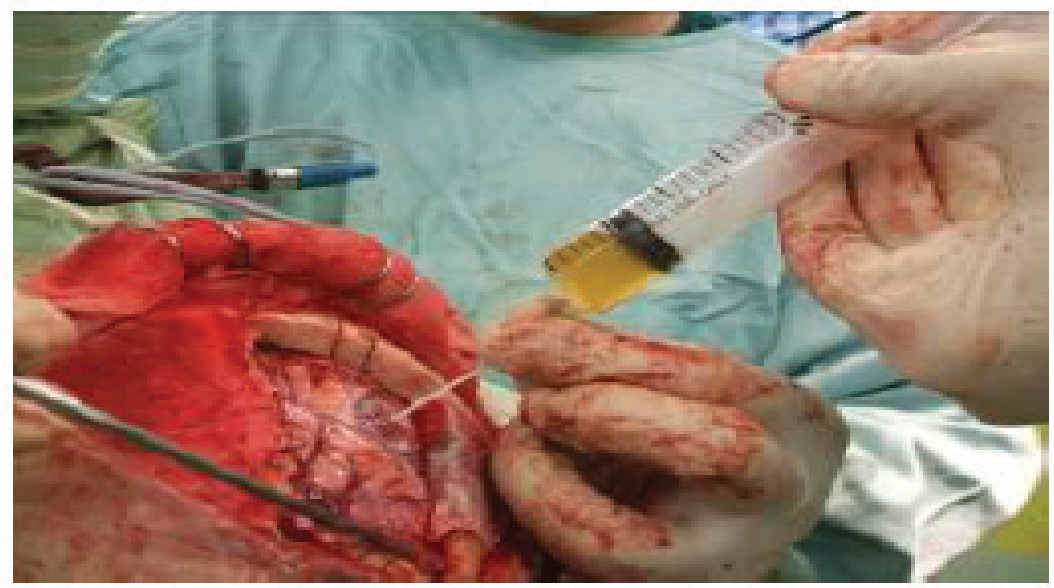

\section{Fig. 5 Macroscopic Features Showed a Cystic Lesion Containing a Dense Proteinaceous Liquid}

changes. ${ }^{8}$ In solid meningiomas, the sensitivity of a CT-scan is virtually $100 \%$ and the specificity is $90 \%$; however, with cystic meningiomas, the diagnosis is made pre-operatively in less than $38 \%$ of cases. ${ }^{2}$ At MRI, cystic meningiomas can be difficult to differentiate from gliomas which partially show enhancement after the injection of contrast media or metastases, due to the presence of cyst which does not enhance and also due to the focal edema. Contrast enhanced
MRI can distinguish cystic walls infiltrated by tumor cells from those formed by gliotic tissue. ${ }^{4}$ Cyst formation in meningiomas is thought to be a resultant of ischemic necrosis, cystic degeneration, intratumoral hemorrhage, trapping of CSF, peritumoral edema into cyst, active secretion by tumor cells, or due to glial reaction and transudation. ${ }^{1}$ The most common location of cystic meningiomas is in the cerebral convexity, particularly in the
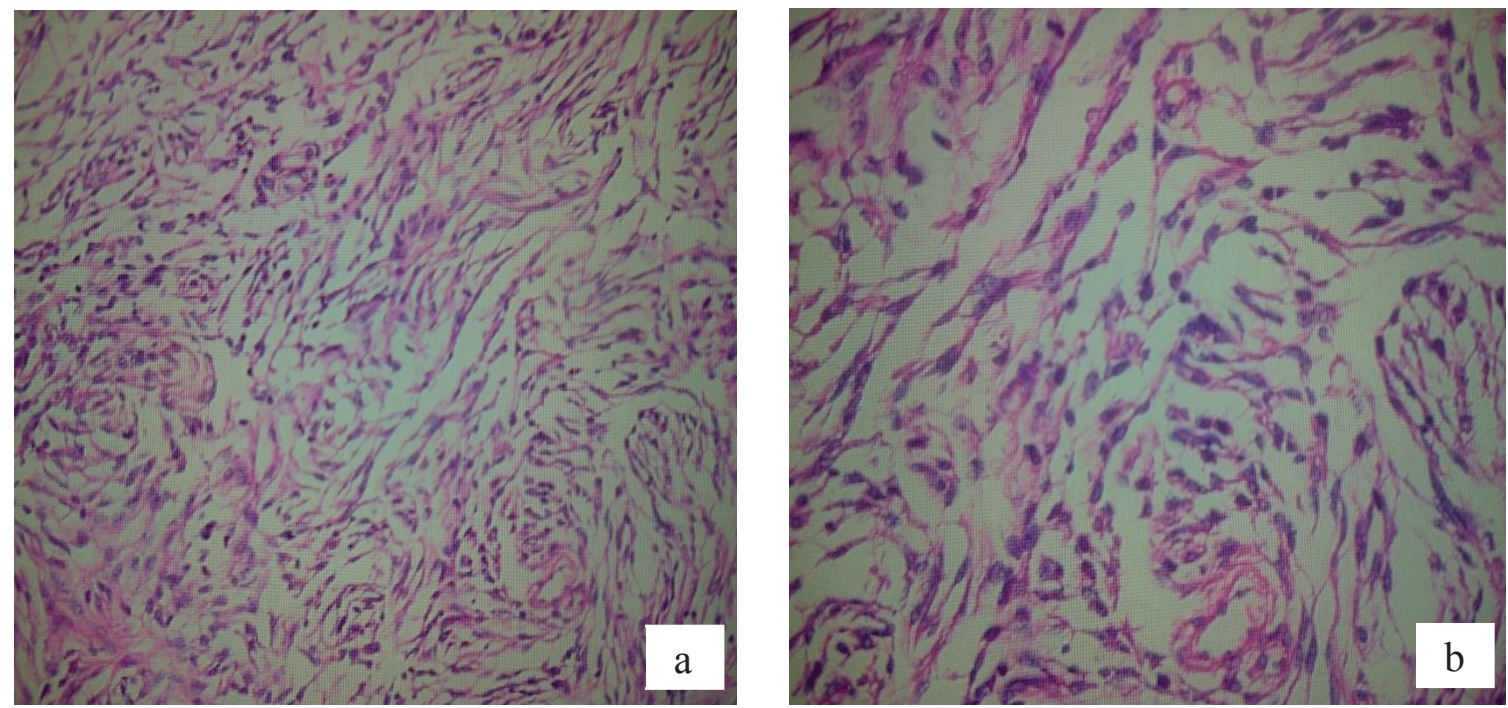

Fig. 6 Pathological Anatomy Revealed a Meningioma 
frontal and parietal regions. The cerebral falx is the second most frequent location. ${ }^{2}$ In this case, location of cystic meningiomas in interhemispheric space make this case very rare.

Cystic meningiomas have been classified into types 1 to 4 . Type I is a central intratumoral cyst, type II is a peripheral intratumoral cyst, type III is a peritumoral cyst in the adjacent brain parenchyma, and type IV is a cyst between the tumor and adjacent brain parenchyma. ${ }^{3,6}$ Based on the fact that the cysts were intratumoral, peritumoral with/without tumor lining cyst wall, and that the cyst is at the brain-tumor interface, the present case was of type 2 .

In terms of therapy for cystic meningiomas, previous studies have shown that total

\section{References}

1. Deb P, Sahni H, Bhatoe HS. Cystic angiomatous meningioma in the cerebellopontine angle mimicking hemangioblastoma. J Cancer Res Ther. 2010;6(4);560-3.

2. Guan TK, Pancharatnam D, Chandran H, Hooi TK, Kumar G, Ganesan D. Infratentorial benign cystic meningioma mimicking a hemangioblastoma radiologically and a lipocytic astrocytoma intraoperatively: a case report. J Med Case Rep. 2013; [cited 2014 Des 3]:[about 4 p.]. Available from: http://www. ncbi.nlm.nih.gov/pmc/articles/PMC3639851/.

3. Hu SL, Li F, Hu R, Cui G, Meng H, Feng H. Atypical histopathologic type of cystic meningioma. Acta Neurochir. 2010;152(1):105-9.

4. Docampo J, Gonzalez N, Vazquez C, Morales C, Gonzalez-Toledo E. Cystic meningioma simulating arachnoid cyst; report of an unusual case. Case Rep Radiol. 2014; [cited 2014 Des 3]:[about 4 p.]. Available from: http://www. ncbi.nlm.nih.gov/pmc/articles/PMC4098774/.

5. Mittal A, Layton KF, Finn SS, Snipes GJ, Opatowsky MJ. Cystic meningioma: unusual imaging appearance of a common intracranial tumor. Proc (Bayl Univ Med Cent). 2010;23(4): removal of tumor is the key for preventing irecurrence. ${ }^{9,10}$ In this present case, the tumor was completely resected. The aim of this report was to highlight an extremely rare location of cystic meningioma for future differential diagnosis.

Imaging studies, including computed tomography and magnetic resonance imaging, had demonstrated a round, enhancing anterior interhemispheric mass, appearing to arise from the falx and caused mild mass effect. The patient underwent a craniotomy and removal of the anterior mass without complication, and her headache and hemiparesis resolved. A pathological examination of the specimen confirmed the diagnosis of meningioma, not glioma. ${ }^{11}$

\section{9-31.}

6. Nauta HJ, Tucker WS, Horsey WJ, Bilbao JM, Gonsalves C. Xanthochromic cysts associated with meningioma. J Neurol Neurosurg Psychiatry. 1979;42(6):529-35.

7. Qiu L, Lui S, Zou L, Yue Q, Gong QY. Lateral ventricular cystic meningioma: 2 rare case reports. Exp Ther Med. 2014;7(5):1393-5.

8. Tabuchi S, Nakajima S. Usefullness of 320-Row area detector computed tomography for the diagnosis of Cystic Falx Meningioma. Case Rep Oncol. 2013;6(2):362-6.

9. Wan X, Jiang B, Ma Z, Wang J, Hou Y, Liu Y. Diagnosis and treatment of cystic meningioma. Zhong Nan Da Xue Xue Bao Yi Xue Ban. 2010; 35(9):1009-12.

10. Ergun T, Torun E, Lakadamyali H. Cystic meningioma showing peripheral rim enhancement without mural nodule. Acta Neurol Belg. 2015;115(3):456-6.

11. Feldman RP, Marcovici A, Suarez M, Goodrich JT. Foreign body granuloma mimicking intracranial meningioma: case report and review of the literature. Neurosurgery. 1999;44(4):855-8. 